
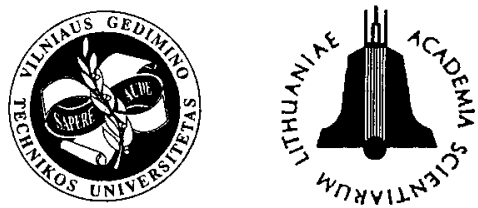

ISSN $1392-3730$

JOURNAL OF CIVIL ENGINEERING AND MANAGEMENT

http:/www.vtu.lt/english/cdition

2002, Vol VIII, No 2, 108-116

\title{
PROBABILISTIC FRAMEWORK FOR ASSESSING RISKS TO STRUCTURES STEMMING FROM ACCIDENTAL EXPLOSIONS
}

\author{
Egidijus R. Vaidogas \\ Dept of Concrete and Masonry Structures, Vilnius Gediminas Technical University, \\ Sauletekio al. 11, LT-2040 Vilnius, Lithuania.E-mail: erv@st.vtu.lt
}

Received 09 May 2001; accepted 05 Febr 2002

\begin{abstract}
In the paper, an attempt is made to formulate a probabilistic framework for an assessment of risk to structures resulting from accidental vapour cloud explosions (VCEs). This framework is based on the general methodology known in the quantitative risk analysis as the classical Bayesian approach to risk assessment. Attention is centred on the estimation of the annual probabilities of potential damages to structural systems exposed to dangers of VCEs. The paper sets up the mathematical problem of assessing risk to structures related to VCEs and, for the most part, contains discussion on the methodological tasks to be solved in this assessment.
\end{abstract}

Keywords: risk, explosion, structure, hazard function, damage, uncertainty, Bayesian approach.

\section{Introduction}

Accidental explosions are dangerous, generally largescale phenomena, and accidents involving such phenomena impose severe consequences, which in many cases include damages to structural systems exposed to the explosions. Industrial activities involving handling, storing and transportation of such combustible materials as liquefied petroleum gas and liquefied natural gas constitute the potentiality of dangerous phenomena called the vapour cloud explosions (VCEs) and the boiling liquid expanding vapour explosions (BLEVEs) [1]. One can say with reasonable confidence that VCEs and BLEVEs are the most probable type of accidental explosions if considered on the scale of the entire industry of a country [2]. The consequences of VCEs and BLEVEs, first of all, loss of life and damage to property have been shown to be severe [3-5].

The likelihood and severity of potential damage to the structural systems immediately exposed to dangers of VCEs and BLEVEs may be assessed most consistently using the methodology of the quantitative risk assessment (QRA) $[4,6]$. An application of QRA in an effort to obtain estimates of risks to a structure coming from a VCEs and BLEVEs requires to widen the conventional use of the structural reliability analysis (SRA) and to agree methods of SRA with the methodology of QRA [7].

In the case of a VCE, the connecting link between the QRA and SRA are mechanical effects (actions) of VCEs endangering the structural system under analysis. A QRA performed for a facility involving the danger of a VCE can yield a probabilistic model of the mechanical effects. With this model, a SRA can be performed and estimates of risks to the structural system in point computed. The need of a combined application of QRA and SRA stems from the fact that there exist considerable uncertainties in the likelihood of imposition of the mechanical effects as well as their characteristics and thus in the possibility and severity of damages to the structural system considered.

The methodology of QRA possesses well-elaborated general approaches to a quantitation of uncertainties related to rare dangerous events [8]. Undoubtedly, a VCE can be considered as such an event, and an application of SRA in the process of a VCE-related risk assessment requires to apply these approaches to both modelling of mechanical effects of VCEs and estimation of the likelihood of structural damages. This requires to construct a probabilistic framework, which allows to express and interpret results of the VCE-related risk assessment in the context of the up-to-day approaches to the quantitation of uncertainties.

The probabilistic framework of the VCE-related risk assessment should start from the definition of risk having the form peculiar to the present-day QRA and furthermore incorporate in the proper way settings up of the problems related to SRA. Such a probabilistic framework seems not to be created up to now despite the fact that various scientific fields have collected a considerable knowledge about the physical phenomenon of VCE and its mechanical effects as well as behaviour of structural systems under explosive loads. 
In the present paper, an attempt is made to formulate the probabilistic framework of the VCE-related risk assessment on the basis of the general methodology known in QRA as the classical Bayesian approach to risk assessment. Attention is centred on the estimation of the annual probabilities of the potential damages to structural systems exposed to dangers of VCEs. The paper is intended as a global setting up of the problem of the VCE-related assessment of the risks to structures and, for the most part, contains discussion on the problems to be solved in the course of this risk assessment.

\section{Questions to be answered}

Risk associated with a VCE can be represented in the standard form called the risk profile [9]. The physical nature of VCE predetermines that many, if not all, of the pairs "outcome - likelihood" in the risk profile will be related to failures of structural components apart from the case when the VCE cannot occur in a built-up area or the structural system being considered is located at a "safe" distance from the region, where the VCE can take place.

A risk profile associated with a VCE can be established for the particular structural system being a subject of an QRA. The set of consequences of this risk profile will contain damages to the structural system and corresponding frequencies of these events. Such a risk profile can be of interest to the owner of the structural system or the regulatory agency which controls the use of the structural system or else the underwriter to which the risk related to a VCE is transferred.

The risk profile related to a particular structural system can be expressed as

$$
\text { Risk } \equiv\left\{\left(F\left(D_{i}\right), L_{D_{i}}\right), i=1,2, \ldots, n_{d}\right\}
$$

where $F\left(D_{i}\right)$ is the frequency (annual probability) of the damage to the system, $D_{i} ; L_{i}$ is the loss suffered in the case of an occurrence of $D_{i}$. In this risk profile, the damage $D_{i}$ is considered as a consequence (random event) and one of these random events, say, $D_{n_{d}}$ means "no" damage.

The definition of risk given by Eq (1) poses two questions of structural nature, namely,

(a) how to establish the spectrum of structural consequences, $D_{1}, D_{2}, \ldots, D_{n_{d}}$;

(b) how to estimate the frequencies $F\left(D_{1}\right), F\left(D_{2}\right), \ldots$ $\ldots, F\left(D_{n_{d}}\right)$.

Both questions are closely related and it is impossible to answer the second question having no answer to the first one.

\section{Categorisation of structural consequences}

An establishing of the spectrum of consequences, $D_{1}, D_{2}, \ldots, D_{n_{d}}$, is governed by the need to classify structural failures, which or combinations of which constitute the damages $D_{i}$. On the other hand, the spectrum of consequences should be established in the way, which allows a clear classification and evaluation of the losses $L_{D_{1}}, L_{D_{2}}, \ldots, L_{D_{n_{d}}}$. A solution of this problem dictates the number of consequences, $n_{p}$. One can concede that number of consequences and structure of the spectrum of consequences is not an unique objective feature of the structural system being analysed, and both the number and the structure can be changed according to the risk management aims.

Establishing the spectrum of consequences is to a great extent an affair of the persons who participate in the decision-making pertaining to evaluation, retention or transfer of the risk arising from the possibility of a VCE. Of course, each spectrum of consequences will always include the desirable state of the structural system under analysis, namely, no damage in case of a VCE. In principle, the spectrum of consequences, $D_{1}, D_{2}, \ldots, D_{n_{d}}$, can be represented as a list of all possible failures which the structural system under analysis may suffer in case of a VCE. However, the owner (underwriter, regulatory agency) will more likely be interested in grouping the structural failures which allows to represent the spectrum of consequences as a set of essentially different undesirable states related to radically different losses suffered in case when the structural system gets into one of the states in consequence of a VCE. For instance, a lessor of an industrial building may be interested in the spectrum of consequences

$D_{1}=$ "building gets into irreparable state",

$D_{2}=$ "building gets into reparable state, long interruption of production process is required",

$D_{3}=$ "building gets into reparable state, short interruption of production process is required",

$D_{4}=$ "minor damages to structural components, no interruption of production process is required",

$D_{5}=$ "no damages to structural components occur, no interruption of production process is required".

The regulator of the government agency concerned with the workplace safety in industrial buildings may demand from the owner of a building to assess risk based on the spectrum of consequences

$D_{1}=$ "people working inside the building are killed or injured in consequence of structural failures caused by a VCE occurring outside the building in point",

$D_{2}=$ "no fatalities or injuries in consequence of structural failures caused by a VCE occurring outside the building in point",

$D_{3}=$ "no structural failures occur in consequence of a VCE".

Finally, the operator of a bare pipeline built in a vicinity of a liquefied natural gas facility may have an 
interest in the spectrum of consequences

$D_{1}=$ "VCE causes mechanical damage to the pipeline with distuption of pipes and release of supplied material",

$D_{2}=$ "VCE causes mechanical damage to the pipeline without disruption of pipes",

$D_{3}=$ "VCE does not cause any mechanical damage to pipeline".

If the consequence $D_{n_{d}}$ means a survival of structural system in case of a VCE, the consequences $D_{1}, D_{2}, \ldots, D_{n_{d}-1}$ can be structural failures of any complexity. In the light of the aforesaid considerations on establishing the spectrum of consequences, $D_{1}, D_{2}, \ldots, D_{n_{d}}$, the definition of each consequence $D_{i}$ $\left(i^{1} n_{d}\right.$ ) is a common task of the risk analyst (structural engineer) involved in the risk assessment process and the person who is going to accept the corresponding potential loss $L_{i}$ or to transfer the risk related to the loss.

The decision which structural failure(s) will be considered as the consequence $D_{i}\left(i \neq n_{d}\right)$ may be dictated by economic reasons or even legal considerations. For instance, the consequence "building gets into an irreparable state due to a VCE" will mean an exceedance of one or several ultimate limit states by the load-bearing structures of the building. At the same time, excessive deformations of its structures, that is, exceedance of one or several serviceability limit states, which do not necessarily lead to the danger of subsequent collapse of the building, may be considered by its owner as such state of his property in which a repair is not justified from economical reasons and a demolition of the damaged building is preferable. The consequence $D_{i}\left(i \neq n_{d}\right)$ may also include all structural failures which, if occur in consequence of a VCE, can endanger people working inside of a building and so impose legal responsibility on the person who runs a production inside.

Once the consequences $D_{i}\left(i \neq n_{d}\right)$ have been preliminary determined, the next step of the risk analysis is to give a precise definition of the random events $D_{i}$ $\left(i \neq n_{d}\right)$ in the context of SRA. This step consists in grouping the limit states, which the structural system being analysed can exceed in case of a VCE. If all the $n_{f}$ identifiable random events of exceedance of limit states in case of a VCE are represented by the set $\left\{S_{1}, S_{2}, \ldots, S_{j}, \ldots, S_{n_{f}}\right\}$, each of the consequences $D_{i}$ $\left(i \neq n_{d}\right)$ can be defined as

$$
D_{i}=\bigcup_{k \in J_{i}} S_{k}
$$

where $S_{j}$ denotes the random event of an exceedance of the limit state $j$ and $J_{i}$ is the subset of values of the index $j$ such that all random events $S_{j}\left(j \in J_{i}\right)$ are relevant to the consequence $D_{i}$. With the definition (2), the conditional probability of a suffering the consequences $D_{i}$ given a VCE is expressed as

$$
P\left(D_{i} \mid A\right)=P\left(\bigcup_{k \in J_{i}} S_{k} \mid A\right)
$$

where $A$ denotes the random event of a VCE. Because the risk profile (1) is usually defined in such a way that the consequences $D_{1}, D_{2}, \ldots, D_{n_{d}}$ are mutually exclusive events, and the union of all $D_{i}$ is the certain event, the definition of the consequences $D_{1}, D_{2}, \ldots, D_{n_{d}}$ must satisfy the condition

$$
\sum_{i=1}^{n_{d}} P\left(D_{i} \mid A\right)=1 .
$$

In terms of the conditional probabilities $P\left(D_{i} \mid A\right)$, the frequencies $F\left(D_{i}\right)$ appearing in the risk profile (1), that is, the frequencies of suffering the consequences $D_{i}$ can be defined as

$$
F\left(D_{i}\right)=F(A) P\left(D_{i} \mid A\right),
$$

provided that the likelihood of a VCE can be modelled in terms of the annual probability (frequency) $F(A)$.

The expression of the likelihood of a VCE in terms of the frequency $F(A)$ agrees with the risk profile definition (1). What is more the form of the profile requires to define the likelihood of the random event $A$ by an annual probability. Such a definition, however, can be inconsistent with the real state of affairs.

In general, the probability of a VCE can increase with time due to a natural wear of the facilities used for handling, storing and transportation of combustible materials which can be accidentally released, vapourised and explode. A time-dependent modelling of the probability of a VCE will automatically change the definition of risk, that is, the probabilities of suffering the consequences, $P\left(D_{i}\right)$, will become time-dependent. An assessment of risk based on the time-dependent analysis is generally more realistic than the one resting on the risk profile (1). The latter, however, can be considered as a simplest definition of the VCE-related risk. A detailing of the estimation of the frequency $F(A)$ and the probabilities $P\left(D_{i} \mid A\right)$ in the framework of the risk assessment based on the risk profile (1) can provide an useful methodological basis for the time-dependent risk assessment.

The following section of the present paper considers how to estimate the frequency $F(A)$ and the probabilities $P\left(D_{i} \mid A\right)$ and thus the frequencies of possible damages (structural failures), $F\left(D_{i}\right)$.

\section{Estimation of frequencies of damages to structure}

\subsection{Conceivable approaches}

The expression of the frequencies of possible damages, $F\left(D_{i}\right)$, given by $\mathrm{Eq}(3)$ is based on the simple definition of conditional probability. The definition (3) states that the estimation problem of these frequencies can be decomposed into two problems, namely, an estimation of the frequency $F(A)$ and an estimation of each of the probabilities $P\left(D_{i} \mid A\right)$. 


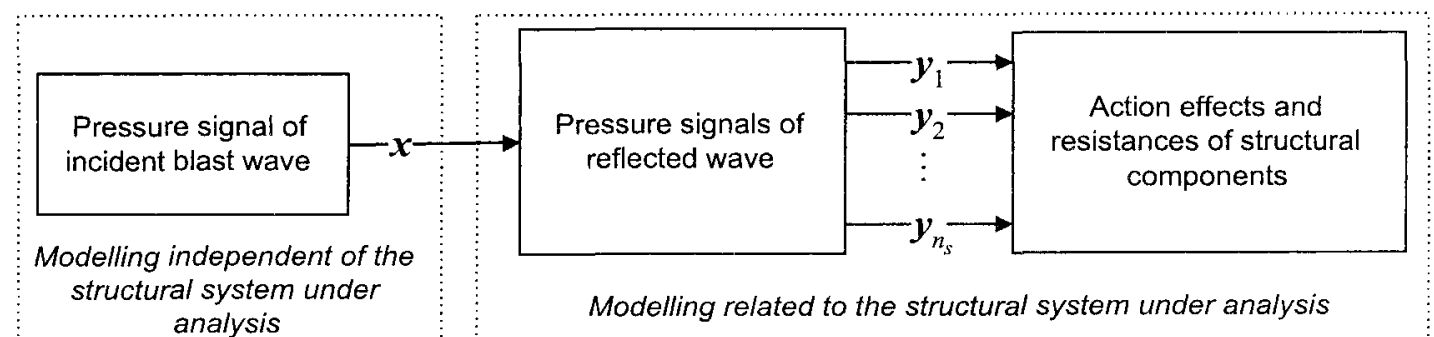

Two stages of the mathematical modelling in the course of an assessing the frequencies of structural failures $(x=$ characteristics of the pressure signal of the incident blast wave; $y_{1}-$ mechanical actions imposed by the blast wave in the $/$ th area of external surface of the structural system under analysis; $l=1,2, \ldots, n_{s}$ )

Although the estimation of annual probabilities and conditional probabilities can be considered as a problem of classical statistics, and confidence intervals of $F(A)$ and $P\left(D_{i} \mid A\right)$ as well as $F\left(D_{i}\right)$ can be computed practically for any amount of data on occurrences of the events $A$ and $D_{i}$, it should be clear from the outset that the estimation based on the approach provided by the classical statistics is virtually impossible. One can adduce at least two arguments for it. Firstly, VCEs, especially detonations of unconfined vapour-air clouds, are rare events even considered on the world-wide scale, and each VCE can be considered as an unique rather than typical event (accident). Secondly, the situation of the exposure of the structural system under analysis to the danger of a VCE is more often than not unique, to say nothing about particular structural components of the system. All this means that in the majority of cases the analyst will be faced with the necessity to estimate the probabilities $F(A)$ and $P\left(D_{i} \mid A\right)$ in the situation of paucity or even absence of suitable accidental data on occurrences of the events $A$ and $D_{i}$.

The situation of paucity or absence of suitable accidental data occurs universally in all assessments of risk stemming from low-frequency high-consequence events. In QRA, two approaches known as "classical Bayesian approach" and "fully Bayesian approach" to risk assessment are suggested to cope with this situation $[8,10]$. As regards data or, more generally, input information in QRA, the approaches lie in a combination of objective statistical data and mathematical models chosen on the basis of experimentation with subjective engineering judgement. The lack of objective information is compensated by subjective judgement or, in other words, "the Bayesian approach[es] does not break down in the absence of large amounts of data" [8]. The use of engineering judgement results in the fact that the final result of QRA, namely, the risk profile is also subjective to a large degree.

In the Bayesian approaches, the concept of probability is used as the analyst's measure of uncertainty or degree of belief. As regards the problem considered in the present paper, an application of one of the Bayesian approaches to risk assessment related to structural con- sequences of VCEs would require to redefine the probabilities $F(A)$ and $P\left(D_{i} \mid A\right)$ according to the principles of this approach. In principle, each of the Bayesian approaches can be applied to assess risk to structures coming from a VCE or, more precisely, to assess the probabilities $F(A)$ and $P\left(D_{i} \mid A\right)$. What will differ when applying the classical and the fully Bayesian approaches is the final form of the risk profile (1) as well as meaning of its components (see the rows 2 and 3 in Table).

In what follows it is considered how to interpret and estimate the probabilities $F(A)$ and $P\left(D_{i} \mid A\right)$ in the framework of the classical Bayesian approach. First a brief look is taken at the problem how to apply principles of the classical Bayesian approach to the choice of the hazard functions characterising the pressure signal of the incident blast wave generated by a VCE (the lefthand rectangle in the flowchart shown in Fig). Then these principles are applied to formulate expressions of the probabilities $P\left(D_{i} \mid A\right)$ in the form allowing to utilise methods of SRA to estimating these probabilities (the middle and right-hand rectangles in Fig).

The flowchart shown in Fig 1 illustrates a possibility of the aforementioned decomposition of the estimation of the frequencies of structural failures, $F\left(D_{i}\right)$. The decomposition is achieved by an in-depth probabilistic modelling of characteristics of three physical phenomena: incident blast wave of a VCE, reflection of the incident wave by the structural system analysed, and response of the structural system to the reflected wave. The in-depth probabilistic modelling consists in a redefinition of the frequencies $F\left(D_{i}\right)$ in the form allowing to apply mathematical models of the phenomena just mentioned and so to express stochastic (aleatory) and epistemic (state-of-knowledge) uncertainties in the phenomena and their models as is done using the classical Bayesian approach.

\subsection{Integration of hazard functions of explosions into estimation of possible damage frequencies}

The frequency (annual probability) of a VCE, $F(A)$ determining together with the probabilities $P\left(D_{i} \mid A\right)$ the frequencies of possible damages, $F\left(D_{i}\right)$, is by itself of 
Representation of the risk profile (1) in three methodological frameworks conceivable in QRA

\begin{tabular}{|c|c|c|}
\hline Kow & $\begin{array}{c}\text { Methodological } \\
\text { framework of QRA }\end{array}$ & Result of QRA \\
\hline 1 & classical statistics & $\begin{array}{l}\qquad\left\{(] F_{i, l b}, F_{i, u b}\left[, L_{D_{i}}\right), i=1,2, \ldots, n_{d}\right\} \\
\text { Here }] F_{i, l b}, F_{i, u b l} \text { is the confidence interval of the frequency of possible damage, } F\left(D_{i}\right) \text {; the true } \\
\text { value of } F\left(D_{i}\right) \text { is unknown and unobservable, however, it is property of the technical system } \\
\text { considered; the risk profile is purely objective }\end{array}$ \\
\hline 2 & $\begin{array}{c}\text { classical Bayesian } \\
\text { approach }\end{array}$ & $\begin{array}{l}\qquad\left\{\left(F_{I L_{D i}}\left(p_{D_{i}} \mid \pi_{I_{D_{D}}}\right), L_{D_{i}}\right), i=1,2, \ldots, n_{d}\right\} \\
\text { Here } F_{I I_{D i}}\left(p_{D i} \mid \pi_{1 I_{D i}}\right) \text { is cumulative distribution function expressing epistemic uncertainty } \\
\text { (subjective degree of believe) in the value of the frequency of possible damage, } F\left(D_{i}\right) \text {; the true } \\
\text { value of } F\left(D_{i}\right) \text { is unknown and unobservable; the risk profile is objective by definition, yet it } \\
\text { contains subjective information }\end{array}$ \\
\hline 3 & $\begin{array}{l}\text { fully Bayesian } \\
\text { approach }\end{array}$ & $\begin{array}{l}\left.\qquad\left\{\left(F_{i}, L_{D_{i}}\right), i=1,2, \ldots, n_{d}\right)\right\} \\
\text { Here } F_{i} \text { is the frequency expressing the epistemic uncertainty (subjective degree of believe) in } \\
\text { the occurrence of the damage } D_{i} \text { during one year; occurrence of the event } D_{i} \text { is observable } \\
\text { event and the frequency } F_{i} \text { is used to express analyst's uncertainty in its occurrence; risk } \\
\text { profile is purely subjective }\end{array}$ \\
\hline
\end{tabular}

no use for predicting the dynamic actions imposed on the structural system under analysis by the VCE and thus for the SRA intended to estimate conditional probabilities of the random events $D_{i}$. The value of $F(A)$ simply expresses the likelihood of an occurrence of a VCE having any intensity in the period of one year.

To carry out the SRA is possible if the potential VCEs are characterised by a more informative mathematical model, namely, a hazard function. Hazard functions, by definition, are used in many applications of QRA to describe adverse physical phenomena in terms of annual exceedance probability versus intensity (eg, [8]). The incident blast wave generated by a VCE can be characterised by a hazard function having the following schematic form [11]:

$$
\begin{gathered}
H_{X}\left(\boldsymbol{x} \mid \pi_{x}, p_{A}\right)=p_{A}\left(1-F_{X}\left(\boldsymbol{x} \mid \pi_{x}\right)\right), \\
x \in R^{n_{x}}, \pi_{x} \in R^{n \pi_{x}},
\end{gathered}
$$

where $x$ is the value of the random vector $X$ having the dimension $n_{x}$ and describing characteristics of the pressure signal of the incident blast wave, angle of approaching the structural system under analysis, etc; $F_{X}\left(\boldsymbol{x} \mid \pi_{x}\right)$ is the cumulative distribution function (c.d.f.) of the random vector $X$ with the vector of parameters, $\pi_{x}$, with the dimensionality $n_{\pi_{x}} ; p_{A}$ is another (shorter) notation of the frequency of a VCE, $F(A)$. The hazard function $H_{X}\left(\boldsymbol{x} \mid \pi_{x}, p_{A}\right)$ expresses the annual probability that at least one of the characteristics of the incident blast wave, that is, at least one component of the vector $\boldsymbol{X}$ will exceed the corresponding component of the vector $\boldsymbol{x}$.

The above definition of the hazard function $H_{X}\left(\boldsymbol{x} \mid \boldsymbol{\pi}_{x}, p_{A}\right)$ states that the frequency of a VCE, $p_{A}$, along with components of the vector $\pi_{x}$ is the parameter of this function. In the light of the classical Bayesian ap- proach, the frequency $p_{A}$ as well as annual probability $H_{X}\left(\boldsymbol{x} \mid \pi_{x}, p_{A}\right)$ should be considered as true but unknown and unobservable values, and some probability distributions used to depict the concentration of analyst's knowledge about the values of $p_{A}$ and $H_{X}\left(x \mid \pi_{x}, p_{A}\right)$.

In the classical Bayesian context, it seems to be natural to express the hazard function $H_{X}($.$) as the "loose"$ family of probability distributions

$$
\begin{gathered}
\left\{F_{\Pi_{A}}\left(p_{A} \mid \pi_{\Pi_{A}}\right) ;\left(F_{X_{k}}\left(x \mid \pi_{x k}\right), p_{k}\right),\right. \\
\left.k=1,2, \ldots, n_{A}\right\}
\end{gathered}
$$

and to apply its components $F_{X_{k}}\left(x \mid \pi_{x k}\right)(k=1,2$, $\left.\ldots, n_{A}\right)$ to the subsequent estimation of the conditional probabilities $P\left(D_{i} \mid A\right)$. In $\mathrm{Eq}(5), \mathrm{P}_{A}$ is the random variable with the c.d.f. $F_{\Pi_{A}}\left(p_{A} \mid \pi_{\Pi_{A}}\right)$ used to model epistemic uncertainty in the true value of the frequency $p_{A} ; F_{X_{k}}\left(x \mid \pi_{x k}\right)\left(k=1,2, \ldots, n_{A}\right)$ is the family of c.d.f.s each expressing the stochastic uncertainty in the values of the random vector $\boldsymbol{X}$; and $p_{k}\left(k=1,2, \ldots, n_{A}\right)$ are weights expressing epistemic the uncertainty in the corresponding c.d.f.s $F_{X_{k}}\left(\boldsymbol{x} \mid \pi_{x k}\right)$. The weights $p_{k}$ are also called the analyst's probability that the $k$ th c.d.f. is true. The sum of all weights $p_{k}$ is, of course, equal to one.

A VCE-related risk assessment in the setting of the classical Bayesian approach requires to express the epistemic uncertainties in true but unknown values of $p_{A}$ and $H_{X}\left(x \mid \pi_{x}, p_{A}\right)$ explicitly, that is, in terms of the distributions $F_{\Pi_{A}}\left(p_{A} \mid \pi_{\Pi_{A}}\right)$ and $p_{k}\left(k=1,2, \ldots, n_{A}\right)$ and to progress the uncertainties with the aim to quantify the epistemic uncertainties in true but unknown values of the frequencies $F\left(D_{i}\right)$. A choice of the c.d.f.s $F_{\Pi_{A}}\left(p_{A} \mid \pi_{\Pi_{A}}\right)$ and $F_{X_{k}}\left(x \mid \pi_{x k}\right)$ as well as assignment of the weights $p_{k}$ is a complicated problem that can be solved by a mathematical modelling of accident courses leading to a 
VCE and based on stochastic simulation [11]. The simulation-based modelling of the accident courses and so the choice of the hazard function $H_{X}(\boldsymbol{x})$ is independent of the structural system being the subject of QRA, apart from several variables describing position and orientation of the structural system in respect of the site, where flammable materials can be released, vapourised and ignited.

The c.d.f.s $F_{X_{k}}\left(\boldsymbol{x} \mid \pi_{x k}\right)$ making up the family (5) are probabilistic models of characteristics of the incident blast wave represented by the random vector $X$. Let us assume that the nature of the structural system under analysis allows one to estimate, with a high accuracy, the conditional probability of the damage $D_{i}$ given the incident blast wave with the characteristics $x$, namely, the probability $P\left(D_{i} \mid \boldsymbol{x}\right)$. With the model $F_{X_{k}}\left(\boldsymbol{x} \mid \pi_{x k}\right)$, the conditional probability of damage $D_{i}$ due to a VCE of any intensity is given by

$$
P\left(D_{i} \mid A_{k}\right)=\int_{\text {all } x} P\left(D_{i} \mid \boldsymbol{x}\right) d F_{X_{k}}\left(\boldsymbol{x} \mid \pi_{x k}\right)
$$

where $A_{k}$ denotes the random event of VCE which characteristics are expressed by the $k$ th c.d.f. $F_{X k}\left(x \mid \pi_{x k}\right)$. If we keep in mind that the c.d.f.s $F_{X_{k}}\left(x \mid \pi_{x k}\right)$ are weighted with the subjective probabilities $p_{k}$ and these probabilities can also be assigned to the corresponding conditional probabilities $P\left(D_{i} \mid A_{k}\right)$, the conditional probability $P\left(D_{i} \mid A\right)$ present in Eq (3) can be expressed as

$$
P\left(D_{i} \mid A\right)=\sum_{k=1}^{n_{d}} P\left(D_{i} \mid A_{k}\right) p_{k} .
$$

The latter expression can be used to simplify the structure of epistemic uncertainties related to the frequencies of structural failures, $F\left(D_{i}\right)$, defined by $\mathrm{Eq}(3)$ and eventually to facilitate the interpretation of the final form of the risk profile (1). If the subjective probabilities $p_{k}$ are averaged out, the remaining epistemic uncertainty in the terms on the right-hand side of Eq (3) is represented only the c.d.f. $F_{\Pi_{A}}\left(p_{A} \mid \pi_{\Pi_{A}}\right)$. Considering that the conditional probability $P\left(D_{i} \mid A\right)$ is a single number (multiplier), and the epistemic uncertainty in the frequency of a VCE, $F(A)$, is expressed by the random variable $\Pi_{A}$, the linear transformation

$$
\Pi_{D i}=\Pi_{A} P\left(D_{i} \mid A\right)
$$

yields the random variable $\Pi_{D i}$ which can be used to express the epistemic uncertainty in the true but unknown and unobservable value of the frequency $F\left(D_{i}\right)$. It is amply clear that to obtain a particular c.d.f. $F_{\Pi_{D i}}\left(p_{D i} \mid \pi_{\Pi_{D i}}\right)$ of the random variable $\Pi_{D i}$, which argument $p_{D_{i}}$ is another (shorter) notation of the frequency $F\left(D_{i}\right)$, given the c.d.f. $F_{\Pi_{A}}\left(p_{A} \mid \pi_{\Pi_{A}}\right)$ is a straightforward problem.

From Eqs (3) and (8) follows that the same c.d.f. $F_{\pi_{A}}\left(p_{A} \mid \pi_{n_{A}}\right)$ will completely determine the epistemic uncertainties in all frequencies $F\left(D_{i}\right)\left(i=1,2, \ldots, n_{d}\right)$. This, of course, is true if (a) the distribution of the un- certainties given by the weights $p_{k}\left(k=1,2, \ldots, n_{A}\right)$ is averaged out to simplify the final form of the risk profile (1), and (b) the epistemic uncertainties in the values of the conditional probabilities $P\left(D_{i} \mid \boldsymbol{x}\right)$ may be disregarded. If the condition (b) is not met and the epistemic uncertainties in the probabilities $P\left(D_{i} \mid \boldsymbol{x}\right)$ will amount to an epistemic uncertainty in the value of the conditional probability $P\left(D_{i} \mid A\right)$, the linear transformation (8) should be replaced by the more general transformation

$$
\Pi_{D i}=\Pi_{A} \Pi_{P D_{i}}
$$

where $\Pi_{P D_{i}}$ is the random variable used to model epistemic uncertainty in the value of the probability $P\left(D_{i} \mid A\right)$.

From the computational standpoint, there is no need to fit probability distributions to the random variables $\Pi_{P D_{i}}\left(i=1,2, \ldots, n_{d}\right)$ and so to use the transformation (9). The epistemic uncertainties in the probabilities $P\left(D_{i} \mid \boldsymbol{x}\right)$ can be progressed through the Eqs (6) and (7) via the stochastic simulation, which will yield samples of the frequency $p_{D_{i}}$ suitable to fit the c.d.f. $F_{\Pi_{D i}}\left(p_{D i} \mid \pi_{\Pi_{D i}}\right)$. Once the progression of uncertainties in the probabilities $P\left(D_{i} \mid \boldsymbol{x}\right)$ has been done and probability distributions of the random variables $\Pi_{D i}$ chosen, the risk profile (1) must be reformulated in line with the principles of the classical Bayesian approach. In the new form of the risk profile, the frequencies $F\left(D_{i}\right)$ must be replaced by the uncertainty distributions $F_{\Pi_{D i}}\left(p_{D i} \mid \pi_{\Pi_{D i}}\right)$ or, alternatively, some percentiles simplifying the risk profile interpretation (see the row 2 in Table).

\subsection{Modelling uncertainties in structural fragility analysis}

In the context of the problem considered in the present paper, the conditional probabilities $P\left(D_{i} \mid x\right)$ may be considered as a point of intersection of QRA and SRA. An evaluation of these probabilities in broad sense includes modelling (prediction) of dynamic loads imposed by the incident blast wave having the characteristics $x$ and, with these dynamic loads, estimation of the probabilities of the damages $D_{i}$ themselves (see the two righthand rectangles in Fig). Each probability $P\left(D_{i} \mid \boldsymbol{x}\right)$ can be considered as the fragility of the structural system under analysis with respect to the incident blast wave having the characteristics $x$ (see the definition of the fragility of structural and mechanical systems used in QRA, for instance, in [12]).

In the view of SRA, the problem of estimating the conditional probabilities $P\left(D_{i} \mid \boldsymbol{x}\right)$ is alleviated by the fact that the accidental actions generated by a VCE are present in the integral expressions of these probabilities as fixed (non-random) arguments of relevant limit state functions. This generally accelerates an evaluation of the integral expressions, what is of great value for an evaluation of the integral expressions of the probabilities $P\left(D_{i} \mid A_{k}\right)$ (see $\mathrm{Eq}(6)$ ). The number of necessary estimations of the con- 
ditional probabilities $P\left(D_{i} \mid \boldsymbol{x}\right)$ in the entire problem of computing the risk profile (1) will in most cases be considerable.

Each estimation of the conditional probabilities $P\left(D_{i} \mid \boldsymbol{x}\right)$ is preceded by a computation of values of accidental actions for given characteristics of the incident blast wave, that is, for given $\boldsymbol{x}$. This can add severity to the estimation, especially if the structural system under analysis has a complex geometry [13]. The accidental actions generated by a VCE are a result of the interaction of incident blast wave with structural system under analysis, and this interaction gives rise to such phenomena as reflection and diffraction of the wave. A VCE can impose a very complex distribution of dynamic loading on external surface of the structural system under analysis [14]. This must be taken into account when formulating particular expressions of the probabilities $P\left(D_{i} \mid x\right)$.

To formulate the integral expression of the conditional probabilities $P\left(D_{i} \mid \boldsymbol{x}\right)$, let us first assume that the entire external surface of the structural system analysed can be divided into $n_{s}$ areas such that mechanical actions of a VCE in each area can be described by the vector $y_{l}$ with the dimensionality $n_{s l}\left(n_{s l} \geq 1\right)$. Assume further that there exist vector-functions

$$
\boldsymbol{y}_{l}=\psi_{l}\left(\boldsymbol{x}, \boldsymbol{\pi}_{\psi l}\right)\left(l=1,2, \ldots, n_{s}\right)
$$

allowing to relate the characteristics of the incident blast wave, $\boldsymbol{x}$, to the mechanical actions imposed by the incident wave on each of the $n_{s}$ areas. The vectors $\pi_{\psi l}$ in the vector-functions $\psi($.$) contain parameters of these$ functions.

If the subset of the vectors $y_{l}$ relevant to the damage $D_{i}$ is represented by the matrix

$$
\mathbf{y}_{i}=\left(y_{1}, y_{2}, \ldots, y_{n_{s i}}\right)\left(n_{s i} \leq n_{s}\right),
$$

elements of this matrix will be arguments of the limit state functions $g_{k}().\left(k=1,2, \ldots, n_{f}\right)$ which are used to define the damage $D_{i}$ in terms of structural mechanical models. Then the definition of $D_{i}$ expressed by Eq (2) can be reformulated as

$$
D_{i}=\bigcup_{k \in J_{i}}\left(g_{k}\left(Z_{i}, \mathbf{y}_{i}\right) \leq 0\right)
$$

where $\boldsymbol{Z}_{i}$ denotes the random vector of the basic variables appearing in the limit state functions $g_{k}().\left(k \in J_{i}\right)$. It is obvious that some components of the vector $Z_{i}$ are normally acting loads imposed on the structural system under analysis at the instant a VCE occurs. With the definition (10), the conditional probability $P\left(D_{i} \mid \boldsymbol{x}\right)$ is expressed as

$$
P\left(D_{i} \mid x\right)=\int_{\text {all } z_{i}} 1\left(z_{i}, \mathbf{y}_{i}\right) d F_{Z_{i}}\left(z_{i} \mid \pi_{z i}\right)
$$

with

$$
\mathbf{I}\left(z_{i}, \mathbf{y}_{i}\right)=\left\{\begin{array}{l}
1 \text { if } \bigcup_{k \in J_{i}}\left(g_{k}\left(z_{i}, \mathbf{y}_{i}\right) \leq 0\right) \\
0 \text { else }
\end{array}\right.
$$

where $F_{z_{i}}\left(z_{i} \mid \pi_{z i}\right)$ is the c.d.f. of the random vector $Z$ with the vector of parameters, $\pi_{z i}$

The integral expression (11) corresponds to the standard definition of the probability of structural failure, and the integral in $\mathrm{Eq}$ (11) can be evaluated using wellinvestigated methods of SRA (eg, see the standard [15]). However, an application of the classical Bayesian approach requires to go beyond the traditional SRA and to ask the question about the epistemic uncertainties in the values of the conditional probabilities $P\left(D_{i} \mid \boldsymbol{x}\right)$. A general answer to this question is already available [10]. Here one can give some comments on the peculiarities of quantitation of the epistemic uncertainties related to mathematical modelling of accidental actions imposed by VCEs.

Epistemic uncertainties can be related to the c.d.f. $F_{Z_{i}}\left(z_{i} \mid \pi_{z_{i}}\right)$, the limit state functions $g_{k}\left(z_{i}, \mathbf{y}_{i}\right)$ and above all else mathematical models relating characteristics of incident blast wave, $\boldsymbol{x}$, with actions imposed by the blast wave and represented by the vectors $\boldsymbol{y}_{l}$. Generally the analyst will have to cope with all these three groups of uncertainties. However, one can expect that the epistemic uncertainties related to the functions $F_{z_{i}}\left(z_{i} \mid \pi_{z i}\right)$ and $g_{k}\left(z_{i}, \mathbf{y}_{i}\right)$ will be negligible or at least substantially lower than those related to the functions $\psi_{l}\left(x, \pi_{\psi l}\right)$.

The dynamic behaviour of the most commonly encountered structures under well-defined explosive loading has been investigated over many years, and realistic mechanical models in the form of the limit state functions $g_{k}\left(z_{i}, \mathbf{y}_{i}\right)$ can be used without explicit quantitative modelling the epistemic uncertainties in the functions $g_{k}\left(z_{i}, \mathbf{y}_{i}\right)$ (see the general reviews given in $[16,17]$ ). Furthermore, components of the vectors $Z_{i}$ model structural characteristics (material properties, dimensions and loads acting at normal conditions) which are usually backed with such amount of statistical data which is sufficient to fit the c.d.f.s $F_{Z_{i}}\left(z_{i} \mid \pi_{z i}\right)$ and to apply them with no regard to related epistemic uncertainties.

Dynamic accidental actions generated by the incident blast wave with characteristics $\boldsymbol{x}$ are another matter. Realistic mathematical models, say, the functions $\psi_{l}\left(\boldsymbol{x}, \pi_{\psi l}\right)$ allowing to predict the accidental actions for given $x$ may be not available at all if the structural system analysed and its environment has a complex geometry. The choice of such mathematical models may require a special experimentation on a reduced model of the structural system [13]. All this can necessitate to take proper account of the epistemic uncertainties in the models $\psi_{l}\left(x, \pi_{\psi l}\right)$. Generally such model uncertainties can be expressed in several ways [18].

As an example one can refer to the case that there is epistemic uncertainty in the parameters of several or all functions $\psi_{l}\left(x, \pi_{\psi l}\right)$ and these uncertainties can be expressed by the corresponding c.d.f.s $F_{\Pi_{\psi t}}\left(\pi_{\psi l} \mid \pi_{\Pi_{\psi t}}\right)$. Then the uncertainty in components of the respective vectors $y_{l}$ can be expressed by the c.d.f. 


$$
\begin{gathered}
F_{Y_{l}}\left(y \mid \pi_{Y_{l}}\right)=P\left(\bigcup_{m=1}^{n_{s l}}\left(Y_{m l} \leq y_{m}\right)\right) \\
=\int_{\Delta_{\psi l}} \psi_{l}\left(\boldsymbol{x}, \pi_{\psi l}\right) d F_{\Pi_{\psi l}}\left(\pi_{\psi l} \mid \pi_{\Pi_{\psi l}}\right)
\end{gathered}
$$

with the integration domains

$$
\Delta_{\psi l}=\left\{\psi_{l}\left(x, \pi_{\psi l}\right) \mid \psi_{m l}\left(x, \pi_{\psi l}\right) \leq y_{m l}, m=1,2, \ldots, n_{s l}\right\}
$$

where $Y_{l}$ is the random vector with componens $Y_{m l}$ used to model uncertainties in the accidental actions resulting from the incident blast wave with the characteristics $\boldsymbol{x}$; $\psi_{m l}\left(x, \pi_{\psi l}\right)$ is the $m$ th component of the vector $\psi_{l}\left(x, \pi_{\psi l}\right)$.

The allowance for the functions $\psi_{l}\left(x, \pi_{\psi i}\right)$ or their parameters $\pi_{\psi l}$ to be uncertain gives rise to an epistemic uncertainty in the limit state functions $g_{k}\left(z_{i}, \mathbf{y}_{i}\right)$ and eventually in the conditional probabilities $P\left(D_{i} \mid \boldsymbol{x}\right)$. This uncertainty can be quantified via the stochastic simulation and progressed upward to the epistemic uncertainties in the frequencies of structural failures, $F\left(D_{i}\right)$.

\section{Concluding remarks}

Establishing and evaluating the VCE-related risk profile enclosing structural consequences of a VCE is generally a complex problem. The main contributor to the complexity are considerable uncertainties in mechanical effects of the VCE. A proper modelling of these uncertainties as well as their progression to the uncertainties in the frequencies of structural failures which can be suffered in consequence of the VCE requires to apply the up-to-date approaches developed in QRA for an uncertainty quantitation.

The general methodology known in QRA as the classical Bayesian approach to risk assessment can be applied to express epistemic uncertainties in the frequencies of the VCE-related structural failures. An application the classical Bayesian approach allows a combined use of objective statistical data and subjective engineering judgement to obtain distributions of the epistemic uncertainties in the frequencies of the structural failures. In other words, the application of the classical Bayesian approach yields the risk profile which contains subjective information. The distributions of epistemic uncertainties depict the concentration of analyst's knowledge about the true but unknown and unobservable values of the frequencies of structural failures.

In principle, one can proceed further and apply the so-called fully Bayesian approach to quantitation of uncertainties in the frequencies of structural failures. In the context of latter approach, the frequencies of structural failures are single numbers expressing the analyst's degree of belief in an occurrence of these failures, that is, the fully Bayesian approach yields a purely subjective risk profile. It has been argued that results which can be obtained applying the fully Bayesian approach are more suitable to the risk-based decision-making than the ones which can yield the classical Bayesian approach [8]. However, the aim of the present paper was to consider the applicability of the classical Bayesian approach to the VCE-related risk assessment.

Within the limits of the classical Bayesian approach, the evaluation of the VCE-related risk profile consists in an evaluation of a set of integral expressions including fragilities of the structural system under analysis with respect to a VCE. The set of these integral expressions can be considered as the probabilistic framework of the VCE-related risk assessment. This framework is the main result of the present paper. A practical evaluation of the risk profile starting from the probabilistic framework suggested and, first of all, a progression of uncertainties within the framework is only possible by extensive application of the stochastic simulation. In principle, the practical evaluation may face serious numerical problems and requires considerable computer time. Broadly speaking, the practical evaluation of the VCE-related risk profile is a challenging and, at the same time, urgent problem. If we recall the catastrophic consequences often caused by VCEs, we can see that it makes sense to solve this problem.

The present paper sets up the probabilistic framework for assessing risk to structures related to VCEs and contains discussion on the methodological tasks to be solved in this assessment. A practical application of the proposed probabilistic framework is to be presented in the author's subsequent papers.

\section{References}

1. Markowski A. S. Quantitative risk analysis for installations of liquefied gases. In: Proc. of ARiZB 2001 (K. T. Kosmowski, Ed.). Gdansk-Gdynia: Politechnika Gdanska, 2001, p. 121-138 (in Polish).

2. Vaidogas E. R. A look at data situation in probabilistic risk analysis as applied to structural systems. Civil Engineering (Statyba), Vol V, No 1, 1999, p. 11-21.

3. Balkema B. J. Vapour cloud explosions - An analysis based on accidents. Part 1. J. of Hazardous Materials. 8 (4), 1984 p. 295-311.

4. Dobiech J., Markowski A. S. Uncertainty representation in modelling the BLEVE effects. In: Proc. of ARiZB 2001 (K. T. Kosmowski, Ed.). Gdansk-Gdynia: Politechnika Gdanska, 2001, p. 219-230 (in Polish).

5. Borisov A. A. et al. Pressure waves forming by detonation and combustion of gas mixtures. The Physics of Combustion and Explosion (Физика горения и взрыва), 21(2), 1985, p. 90-95 (in Russian).

6. Vaidogas E. R. Modelling uncertainties in assessing risk to structures caused by accidental explosions. Civil Engineering (Statyba), Vol VII, No 2, 2001, p. 89-98.

7. Vaidogas E. R. Towards the integration of probabilistic risk analysis with structural analysis. Civil Engineering (Statyba), Vol V, No 3, 1999, p. 183-192. 
8. Aven T., Pörn K. Expressing and interpreting the results of quantitative risk analyses. Review and discussion. Reliability engineering and system safety, 61, 1998, p. 3-10.

9. Kumamoto H., Henley E. J. Probabilistic risk assessment and management for engineers and scientists. 2nd ed. New York: IEEE Press, 1996. $789 \mathrm{p}$.

10. Aven T., Rettedal W. Bayesian frameworks for integrating QRA and SRA Methods. Structural Safety, 20, 1998, p. $155-165$.

11. Vaidogas E. R. Uncertainties related to hazard functions of accidental explosions. Safety, Risk and Reliability Trends in Engineering. Report of int. conf. held in Malta in 2001. Zürich: Safety and Risk in Engineering, 2001, p. $495-500$.

12. Sundararajan (Raj) C., VoTroung V. Probabilistic Structural Mechanics in System and Plant Risk Assessment. In: Probabilistic Structural Mechanics Handbook (C. (Raj) Sundararajan, Ed.). New York: Van Nostrand, Reinhold, 1993, p. $188-210$.
13. Bailly P. et al. Accidental explosions near buildings, methodology of the valuation of the effects. In: Proc. of 6 th int. conf. on structural safety and reliability ICOSSAR'93 (G. I. Shueller etal., Eds), Vol 3, 1994, Rotterdam: Balkema, p. 1929-1932.

14. Watson A.V. The response of civil engineering structures to impulsive loads. In: Structures under shock and impact III. (P. S. Bulson, Ed.). Southampton, Boston: Computational Mechanic Publications, 1994, p. 3-10.

15. ISO 2394: 1998 (E). General principles on reliability for structures. Geneve: ISO, 1998. 73 p.

16. Garas F. K. et al. (Eds.). Abnormal loading on structures. London: Spon Press, 2000. 360 p.

17. Kappos A. J. (Ed.). Dynamic loading and design of structures. London: Spon Press, 2001. 304 p.

18. Zio E., Apostolakis G. Two approaches to model uncertainty quantitation. In: Proc. of ESREL'96 PSAM-III (C. Cacciabune, Ed.). Berlin etc.: Springer, 1996, p. 631636. 